\title{
A GRAPH-THEORETIC CHARACTERIZATION OF THE CORE IN A HOMOGENEOUS GENERALIZED ASSIGNMENT GAME
}

\author{
TADEUSZ SOZAŃSKI \\ Institute of Sociology, Jagiellonian University \\ 52 Grodzka, 31-044 Kraków, Poland \\ E-mail: ussozans@cyf-kr.edu.pl
}

\begin{abstract}
An exchange network is a socioeconomic system in which any two actors are allowed to negotiate and conclude a transaction if and only if their positions - mathematically represented by the points of a connected graph-are joined by a line of this graph. A transaction consists in a bilaterally agreed-on division of a profit pool assigned to a given line. Under the one-exchange rule, every actor is permitted to make no more than one transaction in each negotiation round. Bienenstock and Bonacich ([1]) proposed to represent a one-exchange network with an $n$-person game in characteristic function form. A special case, known as a two-sided assignment game, was studied earlier by Shapley and Shubik ([10]) who proved that the game representing any one-exchange network has a nonempty core if the underlying graph is bipartite. This paper offers a graph-theoretic criterion for the existence of a nonempty core in the game associated with an arbitrary not necessarily bipartite homogeneous one-exchange network where network homogeneity means that every line of the transaction opportunity graph is assigned a profit pool of the same size.
\end{abstract}

1. Exchange networks. While economic theory focuses on exchange processes in free markets where every two actors with complementary interests are allowed to trade with each other, sociologists who invented the concept of an exchange network have always been more interested in studying power and ensuing reward inequality in social systems in which partner choice and bargaining are subject to network restrictions. Since the publication of the special issue of the journal Social Networks (Vol. 14 (1992), Nos. 3-4) "location of power in exchange networks" has become the topic of mathematical investigations which are still going on ([3], [13], [14]) along with experimental work.

2000 Mathematics Subject Classification: Primary 91A06; Secondary 91D30.

Key words and phrases: game theory, game in characteristic function form, assignment game, core, graph theory, exchange network, matching, line independence number, point covering number.

An extended version of this paper with more theorems will appear in Social Networks, Vol. $28(2006)$. 
In this paper, we define an exchange network as an abstract mathematical object made up of three components.

(1) A transaction opportunity graph $G=(N, L)$.

$G$ is a connected undirected graph whose point set $N$ represents the positions occupied by $n$ actors $(n=|N|)$ who are allowed to negotiate and conclude bilateral transactions with the restriction that a deal between the actors in positions $P$ and $Q$ can be made only if $P Q$ is in the line set $L$ of $G$. A line joining $P$ and $Q$, written as $P Q$ or $Q P$ is a couple $\{P, Q\}$ of distinct points. Since no loops are allowed, $L \subset\{\{P, Q\}: P, Q \in N, P \neq Q\}$.

(2) A profit pool network $C$ over $G$.

$C$ is a mapping which assigns to any line $P Q$ in $L$ a number $C_{P Q}>0$ interpreted as the size of a profit pool to be divided between the occupants of positions $P$ and $Q$. The term transaction in a network line $P Q$ refers to any ordered pair $\left(x_{P Q}, x_{Q P}\right)$ of real numbers such that $x_{P Q} \geq 0, x_{Q P} \geq 0$, and $x_{P Q}+x_{Q P}=C_{P Q}, x_{P Q}\left(x_{Q P}\right)$ being the share negotiated by $P(Q)$.

(3) An exchange regime defined as a family $\mathcal{T}$ of subsets of $L$.

Elements of $\mathcal{T}$ will be called transaction sets or trading patterns (Bonacich's term). It is assumed that $\emptyset \in \mathcal{T}$ and for any line $P Q \in L$ there exists a transaction set $T \in \mathcal{T}$ such that $P Q \in T$. That is, the networkwide negotiation process can end up with no transaction and every line has a chance to be the locus of a transaction.

Transaction sets represent all configurations of bilateral agreements which may happen in a single negotiation round in accordance with a given rule which imposes some limitations on the arrangement and number of transactions across the network. The name "exchange regime" and the germ of the idea elaborated by the author come from Friedkin $([7])$.

A transaction set $T$ is called maximal if there is no $U$ in $\mathcal{T}$ such that $T \subset U$ and $T \neq U$. That is, once for every line $P Q$ in $T$ the bargainers in positions $P$ and $Q$ have come to terms, no more transactions may follow, the negotiation round comes to an end, and $P$ and $Q$ get their negotiated shares of $C_{P Q}$.

A cumulative exchange regime is defined by the following condition: any subset $U$ of any $T$ in $\mathcal{T}$ is also in $\mathcal{T}$. Under a cumulative exchange regime, any one-line set $\{P Q\}$ is in $\mathcal{T}$ so that any two actors who have settled on a pool split can safely wait for the end of a round because their payoffs do not depend on transactions subsequently concluded elsewhere in the network.

An exchange regime is called additive if $T \cup U \in \mathcal{T}$ for any two point-disjoint transaction sets $T, U \in \mathcal{T}, T$ and $U$ being point-disjoint if there is no point $P$ such that $P Q \in T$ and $P Q^{\prime} \in U$ for some points $Q$ and $Q^{\prime}$.

To give an example of a cumulative and additive exchange regime, consider the $k$-exchange regime $\mathcal{T}_{k}(G)$ generated by the $k$-exchange rule which permits to every actor to make at most $k$ transactions per round. Clearly, $T \in \mathcal{T}_{k}(G)$ if and only if $T \subset L$ and $\operatorname{deg}_{T}(P) \leq k$ for any $P \in N$ where $\operatorname{deg}_{T}(P)$ stands for the degree of $P$ in the subgraph $(N, T)$ of $G=(N, L)$. Formally, $\operatorname{deg}_{T}(P)=|\{Q: P Q \in T\}|, \operatorname{deg}(P)=\operatorname{deg}_{L}(P)$. 
Experimental research and formal theorizing has so far focused almost exclusively on one-exchange networks, or those with 1-exchange regime $\mathcal{T}_{1}(G) . T \in \mathcal{T}_{1}(G)$ if and only if no two lines in $T$ have a common endpoint. In graph theory (see Chapter 10 in [8]), any subset $T$ of $L$ with this property is called a matching or an independent set of lines.

The structural parameter of $G=(N, L)$ known as the line independence number is defined as

$$
\beta_{1}(G)=\operatorname{Max}\left\{|T|: T \in \mathcal{T}_{1}(G)\right\}
$$

where $|T|$ stands for the cardinality of $T$. A matching $T$ is called optimal if $|T|=\beta_{1}(G)$. Clearly, every optimal matching is maximal (that is, there is no matching $U$ such that $T \subset U$ and $T \neq U$ ), but not conversely.

Maximality with respect to inclusion, as applied to matchings, has so far been given little attention in graph theory. We need this concept to define relations of exclusion and elementary power in the node set of a one-exchange network. We say that $P$ can exclude $Q$ if there exists a maximal matching $T$ which covers $P$ and does not cover $Q$. A subset $M$ of the line set $L$ covers a point $P$ if $P$ is an endpoint of a line in $M$. $P$ has elementary power over $Q$ if $P$ can exclude $Q$ and $Q$ can't exclude $P$.

In this paper, we consider the dual covering relation obtained by reversing the roles of points and lines, namely, we say that a subset $S$ of the node set $N$ covers a line $P Q$ in $L$ if $P \in S$ or $Q \in S$. The structural parameter known as the point covering number, noted $\alpha_{0}(G)$, is defined ([8], Chapter 10) as the smallest number of nodes which cover all lines of $G$. Formally,

$$
\alpha_{0}(G)=\operatorname{Min}\{|S|: S \text { is a point cover of } G\}
$$

where the term point cover refers to any $S \subset N$ covering all lines in $L$.

2. Multiperson games in characteristic function form. An n-person game in characteristic function form is formally defined ([9]) as a couple $(N, v)$ made up of a finite set of players $N=\left\{P_{1}, \ldots, P_{n}\right\}$ and a characteristic function $v$ which assigns a real number $v(S)$ to any subset $S$ of $N$. The subsets of $N$ are called coalitions. The number assigned by $v$ to $S$, called the value (worth) of $S$, is interpreted as the total payoff the members of $S$ can gain independently of whether other players coordinate their actions within the complementary coalition $N-S$, few smaller coalitions, or play each on one's own account. It is convenient to assume that $v(\emptyset)=0$.

Since the games we consider later in this paper have nonnegative individual and collective payoffs, we add the assumption that $v(S) \geq 0$ for any $S$ and adopt the standard interpretation of $v(S)$ as the amount of a divisible good the coalition $S$ can secure for itself through concerted action. This quantity can be seen as a "cake" from which each coalition member receives a portion provided that all players in $S$ agree to coalesce and are able to settle on how to divide the "cake" among themselves. As regards the actors' goal-orientation, it is assumed that each player wants to gain as much as possible of the good in question and considers every potential coalition solely as a means to this end.

A game $(N, v)$ is called superadditive if $v\left(S \cup S^{\prime}\right) \geq v(S)+v\left(S^{\prime}\right)$ for any two disjoint coalitions $S$ and $S^{\prime}$. Superadditivity is often included in the definition of an $n$-person game in characteristic function form. For any superadditive game, we define a payoff 
vector as a sequence $x=\left(x_{1}, \ldots, x_{n}\right)$ of real numbers such that

$$
x_{i} \geq 0 \text { for } i=1, \ldots, n \text { and } x_{1}+\cdots+x_{n} \leq v(N)
$$

where $x_{i}$ is the number of resource units earned by player $P_{i}$ at the end of the game. By assumption, all payoffs are nonnegative and can be jointly paid from the pool allocated to the grand coalition $N$.

We say that a payoff vector $x$ is feasible for a coalition $S$ if $\sum(S, x) \leq v(S)$ where $\sum(S, x)$ stands for the total payoff of the members of $S$. By definition, every payoff vector is feasible for $N$.

We also assume that every time the game is played there arises a coalition structure $\left\{S_{1}, \ldots, S_{k}\right\}$ which is a partition of $N$ into nonempty, pairwise disjoint subsets whose union is $N$. The coalition structures $\left\{\left\{P_{1}\right\}, \ldots,\left\{P_{n}\right\}\right\}$ and $\{N\}$ represent two simplest contrasting ways of playing the game: the actors fail to create any alliance or all ally to form the grand coalition.

Feasibility of $x$ for a coalition structure $\left\{S_{1}, \ldots, S_{k}\right\}$ is defined by the condition: $\sum\left(S_{j}, x\right)=v\left(S_{j}\right)$ for $j=1, \ldots, k$. The pair made up of a payoff vector and a coalition structure for which the payoff vector is feasible is called payoff configuration.

The condition by which we defined the feasibility of $x$ for a coalition structure is stronger than the requirement that $x$ is feasible for every $S_{j}$. However, the payoff vectors such that $\sum\left(S_{j}, x\right)<v\left(S_{j}\right)$ for some $S_{j}$ need not be counted as outcomes of a superadditive game, since every payoff vector $x$ with this property can be replaced by an $x^{\prime}$ such that $\sum\left(S_{j}, x^{\prime}\right)=v\left(S_{j}\right)$ for all $j$, and $x_{i}^{\prime} \geq x_{i}$ for every player $P_{i}$. To obtain $x^{\prime}$, each undistributed surplus $v\left(S_{j}\right)-\sum\left(S_{j}, x\right)$ is divided evenly among the members of $S_{j}$. Then, without any loss to the members of other coalitions, all players in $S_{j}$ are better off $\left(x_{i}^{\prime}>x_{i}\right)$. The inequality $\sum\left(N, x^{\prime}\right) \leq v(N)$ which is required of any payoff vector is met by $x^{\prime}$ in virtue of superadditivity, namely, $v(N) \geq v\left(S_{1}\right)+\cdots+v\left(S_{k}\right)$.

A payoff vector $x$ is feasible if there exists at least one coalition structure for which $x$ is feasible. Feasible payoff vectors form the most comprehensive "space of solutions" of a game in characteristic function form.

An additional restriction which is usually imposed on the outcomes of a superadditive game is the condition of individual rationality:

$$
x_{i} \geq v\left(\left\{P_{i}\right\}\right) \quad \text { for all } i .
$$

This natural postulate means that no player will accept a lower payoff than the payoff he can safely gain by acting as a single actor coalition.

The group (collective) rationality condition is given by the equation

$$
\sum(N, x)=v(N) \text {. }
$$

The equation means that $x$ is feasible for the coalition structure $\{N\}$ made up of one coalition containing all players.

For any superadditive game $(N, v)$ we have $v(N) \geq v\left(\left\{P_{1}\right\}\right)+\cdots+v\left(\left\{P_{n}\right\}\right)$. Superadditive games for which $v\left(\left\{P_{1}\right\}\right)+\cdots+v\left(\left\{P_{n}\right\}\right)=v(N)$ are called inessential. Then, for any $S, v(S)$ is the sum of $v\left(\left\{P_{i}\right\}\right)$ over all $P_{i} \in S$, which implies in turn that $x_{i}=v\left(\left\{P_{i}\right\}\right)$, $i=1, \ldots, n$, is the only feasible, individually rational payoff vector. Since in these cir- 
cumstances the players gain nothing by forming larger coalitions, the theory focuses on essential games, or those satisfying the inequality $v(N)>v\left(\left\{P_{1}\right\}\right)+\cdots+v\left(\left\{P_{n}\right\}\right)$.

For essential games, the set of imputations (payoff vectors which meet the conditions of individual and group rationality) is usually too large so that further restrictions need to be imposed on theoretically predicted outcomes of the game. The condition of coalition rationality has the form

$$
\sum(S, x) \geq v(S) \quad \text { for all } S \subset N .
$$

The set $C r(v)$ of coalitionally rational payoff vectors is called the core of the game $(N, v)$. Coalition rationality implies that $x_{i} \geq v\left(\left\{P_{i}\right\}\right)$ for $i=1, \ldots, n$. Thus, all payoff vectors in the core satisfy the condition of individual rationality. Group rationality condition $\sum(N, x)=v(N)$ is also met because the inequality $\sum(N, x) \leq v(N)$ is assumed for every payoff vector and the inequality $\sum(N, x) \geq v(N)$ results from coalition rationality. Thus, the core can be equivalently defined as the set of all coalitionally rational imputations $([9])$.

Group rationality condition implies that every payoff vector in the core is feasible for the coalition structure $\{N\}$. Below we prove a theorem which specifies the range of coalition structures for which every payoff vector in $\operatorname{Cr}(v)$ is feasible. We say that a coalition structure $\mathcal{S}=\left\{S_{1}, \ldots, S_{k}\right\}$ is optimal if it satisfies the condition

$$
v\left(S_{1}\right)+\cdots+v\left(S_{k}\right)=v(N) .
$$

THEOREM 2.1. Any payoff vector $x$ from the core of the game $(N, v) x$ is feasible for a coalition structure $\mathcal{S}$ if and only if $\mathcal{S}$ is optimal.

Proof. Assume that $x \in C r(v)$. Consider an optimal coalition structure $\mathcal{S}=\left\{S_{1}, \ldots, S_{k}\right\}$. We show that the optimality condition implies that $x$ is feasible for $\mathcal{S}$. By applying the coalition rationality condition to every $S_{j}$ and adding up the right and left hand sides of the respective inequalities, we arrive at the inequality $\sum_{j} \sum\left(S_{j}, x\right) \geq \sum_{j} v\left(S_{j}\right)$, or $\sum(N, x) \geq v(N)$. Since $\sum(N, x)=v(N)$, none of the inequalities $\sum\left(S_{j}, x\right) \geq v\left(S_{j}\right)$ can be sharp, which means that $x$ is feasible for $\mathcal{S}$. The proof of the necessity of the condition $\sum_{j} v\left(S_{j}\right)=v(N)$ for the feasibility of $x$ for the coalition structure $\left\{S_{1}, \ldots, S_{k}\right\}$ is even simpler: $v(N)=\sum(N, x)=\sum\left(S_{1}, x\right)+\cdots+\sum\left(S_{k}, x\right)=v\left(S_{1}\right)+\cdots+v\left(S_{k}\right)$ by group rationality and feasibility of $x$.

Theorem 2.1 will help us to derive many results for the class of games we define in the next section after Bienenstock and Bonacich $([1])$ who were first to apply game theory to exchange networks.

3. The $n$-person game associated with an exchange network. The n-person game associated with an exchange network over $G=(N, L)$ with an exchange regime $\mathcal{T}$ and a profit pool network $C$ is defined as $\left(N, v_{C, \mathcal{T}}\right)$ where $N$ - the set of network nodesbecomes the set of players, and the value of the characteristic function $v_{C, \mathcal{T}}$ for any coalition $S \subset N$ is given by the formula

$$
v_{C, \mathcal{T}}(S)=\operatorname{Max}\left\{\sum(T, C): T \in \mathcal{T} ; \text { if } P Q \in T, \text { then } P \in S \text { and } Q \in S\right\}
$$

where $\sum(T, C)$ stands for the sum of $C_{P Q}$ over all $P Q$ in $T\left(\sum(\emptyset, C)=0\right)$. The maximum 
of $\sum(T, C)$ is taken over all transaction sets $T$ such that both endpoints of any line in $T$ lie in $S$. Note that under any exchange regime

$$
v_{C, \mathcal{T}}(\{P\})=0 .
$$

Assume that $\mathcal{T}$ is cumulative. Then $\{P Q\} \in \mathcal{T}$. Since $\{P Q\}$ is the only transaction set meeting the condition given in the definition of $v_{C, \mathcal{T}}(S)$ for $S=\{P, Q\}$, we have

$$
v_{C, \mathcal{T}}(\{P, Q\})=C_{P Q} \quad \text { for any } P Q \in L ; \quad v_{C, \mathcal{T}}(\{P, Q\})=0 \quad \text { for any } P Q \notin L .
$$

The values of $v_{C, \mathcal{T}}$ for triplets and larger coalitions depend on a concrete exchange regime. For now, more specific results are available solely for one-exchange networks, Thus, we must conclude this section with just one general theorem.

THEOREM 3.1. If the exchange regime in an exchange network is cumulative and additive, then the associated game is superadditive and essential.

The proof of Theorem 3.1 is an immediate consequence of the definitions, so it can be omitted.

By associating the characteristic function game $\left(N, v_{C, \mathcal{T}}\right)$ with an exchange network $(N, L, C, \mathcal{T})$ we obtain a functor from the category of exchange networks to the category of superadditive and essential games. The structuralist methodology of mathematics necessitates defining for any category at least the class of isomorphisms. In general, an isomorphism of two mathematical objects with base sets $N$ and $N^{\prime}$ and structures $S$ and $S^{\prime}$ of the same type is a 1-1 mapping $\pi$ of $N$ onto $N^{\prime}$ which induces a 1-1 correspondence between $S$ and $S^{\prime}$. In particular, $\pi$ is an isomorphism of two exchange networks $(N, L, C, \mathcal{T})$ and $\left(N^{\prime}, L^{\prime}, C^{\prime}, \mathcal{T}^{\prime}\right)$ if: (1) $\pi$ is an isomorphism of graphs $(N, L)$ and $\left(N^{\prime}, L^{\prime}\right)$, that is, for any $P, Q \in N$ such that $P \neq Q, P Q \in L$ if and only if $\pi(P) \pi(Q) \in L^{\prime} ;(2)$ There exists a number $s>0$ such that for any line $P Q$ in $L$ we have $C_{\pi(P) \pi(Q)}^{\prime}=s C_{P Q}$; (3) For any $T \subset L, T \in \mathcal{T}$ if and only if $\pi(T) \in \mathcal{T}^{\prime}$ where $\pi(T)=\{\pi(P) \pi(Q): P Q \in T\}$.

Notice that every automorphism (the term "automorphism" is used when two mathematical objects are identical) of a graph $G=(N, L)$ automatically satisfies condition 3 under the $k$-exchange regime $\mathcal{T}_{k}(G)$.

Two characteristic function games $(N, v)$ and $\left(N^{\prime}, v^{\prime}\right)$ are isomorphic under $\pi$ if there exists a scaling factor $s>0$ such that $v^{\prime}(\pi(S))=s v(S)$ for any $S \subset N$. Clearly, every isomorphism of two exchange networks is also an isomorphism of the games associated with them. Thus, the functor from the category of exchange networks to the category of characteristic function games - which is defined for objects as $(N, L, C, \mathcal{T}) \rightarrow\left(N, v_{C, \mathcal{T}}\right)$ becomes the assignment $\pi \rightarrow \pi$ for isomorphisms.

Let $(N, v)$ be a superadditive essential game with nonnegative values. Does there exist an exchange network $(N, L, C, \mathcal{T})$ with cumulative and additive exchange regime $\mathcal{T}$ such that $v=v_{C, \mathcal{T}}$ ? For now we can only state an obvious necessary condition: $v(\{P\})=0$ for any $P \in N$, and every $S$ such that $v(S)>0$ contains some points $P$ and $Q$ such that $v(\{P, Q\})>0$.

4. Generalized assignment games. Let $v_{C}=v_{C, \mathcal{T}_{1}(G)}$ denote the characteristic function of the $n$-person game associated with a one-exchange network over $G=(N, L)$. To compute $v_{C}(S)$ for any $S \subset N$, one needs to determine the maximum of $\sum(T, C)$ across 
all matchings which consist of lines having both endpoints in $S$. The subset of $\mathcal{T}_{1}(G)$ made up of these matchings coincides with $\mathcal{T}_{1}\left(G_{S}\right)$ where $G_{S}=\left(S, L_{S}\right)$ is the subgraph of $G=(N, L)$ generated by $S$ ( $L_{S}$ stands for the subset of $L$ made up of all lines with both endpoints in $S$ ).

A special case of the game $\left(N, v_{C}\right)$ known as a two-sided assignment game is obtained by assuming that the transaction opportunity graph is bipartite, that is, $N$ is the union of two nonempty, disjoint sets $N_{1}$ and $N_{2}$ such that every line in $L$ has one point in $N_{1}$ and the other point in $N_{2}$.

Under a common economic interpretation, the members of $N_{1}$ are potential sellers and the members of $N_{2}$ are potential buyers of, say, used cars. Every seller (he) and every buyer (she) aims at maximizing his/her profit from a bilateral transaction with a member of the opposite class. A seller's profit is the difference between the negotiated price and the minimum price acceptable to him; a buyer's profit is the difference between maximum price she would pay for the car offered by a given seller and the negotiated price. If the minimum price of a seller $P$ is lower than the maximum price of a buyer $Q$, then the two parties can bargain over the division of the difference between the two prices. Assuming that every seller has and every buyer needs only one car, one can model this simple market as a bipartite one-exchange network, and use its game representation to predict an outcome of the bargaining process.

Shapley and Shubik ([10]) who were first to study two-sided assignment games proved that every game of the kind has a nonempty core (see [9], 221-223; [11], Chapter 8). They showed also that the core determines an "assignment" of buyers to sellers (hence the name "assignment game").

The concept defined by Shapley and Shubik was generalized by Bienenstock and Bonacich ([1]) whose later papers ([2], [3], [4], [5], [6]) provided further results on the existence and shape of the core for the games associated with one-exchange networks. I propose to call these games generalized assignment games. The term "the BienenstockBonacich game" will be used interchangeably.

This paper offers a graph-theoretic characterization of the core for the games associated with homogeneous one-exchange networks, or those in which every line is assigned a profit pool of the same size, that is, for any $P Q \in L, C_{P Q}=C_{0}$ for some $C_{0}>0$. Since the context makes confusion impossible, the letter $C$ will be used to denote both the profit pool matrix and the constant pool size. The choice of $C>0$ affects only the scale for measuring the value of each coalition.

It is not difficult to verify that the characteristic function of a homogeneous generalized assignment game is given by the following formula

$$
v_{C}(S)=C \beta_{1}\left(G_{S}\right)
$$

which holds for any subset $S$ of $N$. In particular, we have $v_{C}(N)=C \beta_{1}(G)$.

All terms introduced in Section 2 for characteristic function games can be applied to generalized assignment games. However, if such a representation of a one-exchange network is to be a tool for analyzing the network itself, then one needs to study those "solutions" of the network game which are compatible with the assumption that the networkwide payoff allocation should arise from two-party transactions. 
The network-oriented approach prompts the following definition. A payoff vector $x$ is said to be feasible for a matching $T$ if $x$ is feasible for the coalition structure determined by $T$, that is, the coalition structure made up of two-player coalitions coinciding with lines in $T$ and of single-player coalitions corresponding to points not covered by $T$. Similarly, we call $x$ a network-feasible payoff vector if $x$ is feasible for some matching $T$.

Once the nodes of an exchange network and the players of the associated game have been noted $P_{1}, \ldots, P_{n}$ we can represent the profit pool network $C$ over $G=(N, L)$ as an $n \times n$ matrix $C=\left(C_{i j}\right)$ where $C_{i j}=C_{P_{i} P_{j}}$ for $P_{i} P_{j} \in L$ and $C_{i j}=0$ for $P_{i} P_{j} \notin L$. The $C$ matrix uniquely determines the adjacency matrix $G=\left(G_{i j}\right)$ of the transaction opportunity graph: $G_{i j}=1$ if $C_{i j}>0, G_{i j}=0$ if $C_{i j}=0$.

A simple consequence of network feasibility is that $P_{i}$ 's payoff $x_{i}$ in the BienenstockBonacich game can be identified with the number of profit points $P_{i}$ gets in a transaction with one of its neighbors in $G$, formally, $x_{i} \leq C_{i j}$ for some node $P_{j}$ such that $P_{i} P_{j} \in L$.

A matching $T$ is called network-optimal if $\sum(T, C)=v_{C}(N)$. In a homogeneous one-exchange network, $v_{C}(N)=C \beta_{1}(G)$ and $\sum(T, C)=C|T|$ for every matching $T$, so that $T$ is network-optimal if and only if $|T|=\beta_{1}(G)$, that is, network-optimality reduces to optimality defined in Section 1 . The theorem which follows shows how network-optimal matchings are related to the core of a generalized assignment game.

THEOREM 4.1. If a payoff vector $x$ is in the core of a generalized assignment game, then $x$ is feasible for a matching $T$ if and only if $T$ is network-optimal.

Proof. Let $\left\{S_{1}, \ldots, S_{k}\right\}$ be the coalition structure generated by a matching $T$, that is, $S_{j}=\{P, Q\}$ for some $P Q$ in $T$ or $S_{j}=\{P\}$ for some $P$. Since $v_{C}(\{P, Q\})=C_{P Q}$ for any $P Q$ in $T$ and $v_{C}(\{P\})=0$ for any $P$, we have $v_{C}\left(S_{1}\right)+\cdots+v_{C}\left(S_{k}\right)=\sum(T, C)$. As a consequence, the conditions $\sum(T, C)=v_{C}(N)$ and $v_{C}\left(S_{1}\right)+\cdots+v_{C}\left(S_{k}\right)=v_{C}(N)$ are equivalent, but the latter was given in Theorem 2.1 as a necessary and sufficient condition for the feasibility of $x \in C r\left(v_{C}\right)$ for $\left\{S_{1}, \ldots, S_{k}\right\}$.

5. The core of a generalized assignment game. Unlike the Shapley value, the core can be empty, which may be a serious problem if one resorts to the game representation of an exchange network to predict results of the negotiation process.

Consider the Triad network with 3 positions $A_{1}, A_{2}, A_{3}$ and 3 lines $A_{1} A_{2}, A_{1} A_{3}$ and $A_{2} A_{3}$, and assume that $C_{12}=\operatorname{Max}\left\{C_{12}, C_{13}, C_{23}\right\}$ so that $v_{C}(N)=C_{12}$. Let $x=$ $\left(x_{1}, x_{2}, x_{3}\right)$ be a payoff vector. If $x \in \operatorname{Cr}\left(v_{C}\right)$, then $x$ is feasible for the network-optimal matching $\left\{A_{1} A_{2}\right\}$, which means that $x_{1}+x_{2}=C_{12}$ and $x_{3}=0$. By applying the coalition rationality condition to $\left\{A_{1}, A_{3}\right\}$ and $\left\{A_{2}, A_{3}\right\}$, we arrive at the inequalities $x_{1} \geq C_{13}$ and $x_{2} \geq C_{23}$ which imply in turn that $C_{12} \geq C_{13}+C_{23}$. Therefore, the latter inequality is the necessary condition of the existence of a nonempty core for $v_{C}$. Clearly, it is a sufficient condition as well. It is not met if $C_{12}=C_{13}=C_{23}$. Thus, the homogeneous Triad is the simplest one-exchange network for which the Bienenstock-Bonacich game has an empty core.

The game associated with a one-exchange network over the 3-node graph of the form $B_{1}-A-B_{2}$ has a nonempty core under any weights assigned to $A B_{1}$ and $A B_{2}$. The core reduces to exactly one payoff vector such that $x_{B_{1}}=x_{B_{2}}=0$ and $x_{A}=\operatorname{Max}\left\{C_{A B_{1}}, C_{A B_{2}}\right\}$. 
Although such an extreme imbalance of benefits rarely occurs in the games played by experimental subjects, the core reflects structural power that the nonexcludable position $A$ has over its excludable neighbors $B_{1}$ and $B_{2}$.

Let $P$ be a node of a one-exchange network $G=(N, L)$. We say that $P$ is excludable if there exists a maximal matching $T$ which does not cover $P$. $P$ is called nonexcludable if it is covered by all maximal elements in $\mathcal{T}_{1}(G)$. The game-theoretic counterpart of excludability is introduced by the following definition: $P$ is game-excludable if there exists a network-optimal matching $T$ which does not cover $P$. Since every network-optimal matching is maximal, every game-excludable point is excludable. The converse is not true, as there exist excludable points that are not game-excludable. To give an example, consider the homogeneous one-exchange network with transaction opportunity graph drawn in the form $B_{1}-A_{1}-A_{2}-B_{2}$ and labeled 4-Chain or 4-Line. Positions $B_{1}$ and $B_{2}$ are excludable - they are not covered by the maximal matching $\left\{A_{1} A_{2}\right\}$-yet they are game-nonexcludable because they are covered by the matching $\left\{A_{1} B_{1}, A_{2} B_{2}\right\}$ which is the only optimal matching in this network.

Theorem 5.1 implies that game-excludable positions gain nothing if the network game ends up with an outcome in the core.

THEOREM 5.1. If a payoff vector $x$ is feasible for all network-optimal matchings, then $x_{i}=0$ for every game-excludable point $P_{i}$.

The proof of Theorem 5.1 is straightforward. Let $\mathrm{T}$ be an network-optimal matching which does not cover $P_{i}$. The feasibility of $x$ for $T$ implies that $x_{i}=v_{C}\left(\left\{P_{i}\right\}\right)=0$. In particular, if $x \in \operatorname{Cr}\left(v_{C}\right)$, then $x_{i}=0$ for any game-excludable $P_{i}$.

The property of game-excludability can be defined for any superadditive game $(N, v)$ by the following statement: a coalition $S \subset N$ is game-excludable if $v(N)=v(N-S)$, in other words, the group does not need the participation of the members of $S$ to achieve the maximum possible collective profit. A player $P \in N$ is said to be game-excludable if coalition $\{P\}$ is game-excludable. The generalization of Theorem 5.1 takes the following form: If a payoff vector $x$ is feasible for all optimal coalition structures in a superadditive game $(N, v)$ and coalition $S$ is game-excludable, then $x_{i}=0$ for all $P_{i}$ in $S$. To prove this fact, notice that $v(N) \geq v(N-S)+v(S), v(N) \geq v(N)+v(S), 0 \geq v(S)$, and $v(S)=0$, in virtue of superadditivity, game-excludability of $S$, and the assumption, made in Section 2 , that a characteristic function takes nonnegative values. Since $v(S)+v(N-S)=v(N)$, the coalition structure $\{S, N-S\}$ is optimal. The feasibility of $x$ for this coalition structure means that $\sum(S, x)=v(S)=0$. Since $x_{i} \geq 0$, by assumption, there must be $x_{i}=0$ for any $P_{i} \in S$.

There are many homogeneous one-exchange networks in which all positions are gameexcludable, the homogeneous Triad being the smallest example. Theorem 5.1 implies that the game associated with every network of the kind is coreless (that is, its core is empty). Indeed, if every position $P_{i}$ is game-excludable and $x \in \operatorname{Cr}\left(v_{C}\right)$, then $x_{i}=0$ for all $i$ so that $\sum(N, x)=0$, which contradicts the group rationality condition $\sum(N, x)=$ $v_{C}(N)>0$.

Coreless homogeneous one-exchange networks can contain game-nonexcludable nodes. The smallest example can be drawn as a "triangle with a two-line tail" (the chain 
$A-C-D$ is added to Triad with nodes relabeled as $\left.A, B_{1}, B_{2}\right)$. In this network, node $C$ is nonexcludable, so it is also game-nonexcludable.

In coreless generalized assignment games, game-excludable players are not always doomed to being "exploited" by their game-nonexcludable neighbors. Two game-excludable players can be connected with each other, which gives them the opportunity to evenly split the pool between themselves. However, if a Bienenstock-Bonacich game has a nonempty core, the situation of game-excludable players is much worse: their only potential partners are game-nonexcludable. This results from the following theorem.

THEOREM 5.2. If a generalized assignment game has a nonempty core, then no two game-excludable nodes are tied with each other in the network.

Proof. Assume that there exists a payoff vector $x$ in $\operatorname{Cr}\left(v_{C}\right)$. Theorem 5.1 implies that $x_{i}=0$ and $x_{j}=0$ for any two game-excludable nodes $P_{i}$ and $P_{j}$. If line $P_{i} P_{j}$ were in $G$, then $0=x_{i}+x_{j} \geq v_{C}\left(\left\{P_{i}, P_{j}\right\}\right)=C_{i j}>0$, which is a contradiction.

The two subsequent theorems were first demonstrated by Bonacich and Bienenstock ([5], [6]). Theorem 5.3 simplifies the verification of the coalition rationality condition, namely, it suffices to consider only the dyadic coalitions in which utility transfer is possible. If all connected dyads are coalitionally rational, then so is every larger coalition.

THEOREM 5.3. An $n$-dimensional vector $x$ is in the core of the game $\left(N, v_{C}\right)$ associated with the one-exchange network $C$ over $G=(N, L)$ if and only if $x$ satisfies the following three conditions:

(1) $x_{i} \geq 0$ for all $P_{i} \in N$;

(2) $x_{i}+x_{j} \geq C_{i j}$ for all $P_{i} P_{j} \in L$;

(3) $x_{1}+\cdots+x_{n}=v_{C}(N)$.

Proof. The only thing we have to demonstrate is that condition 2 implies the rationality of any $S \subset N$ such that $|S|>2$. Let $T$ be a matching in $G_{S}$ such that $\sum(T, C)=v_{C}(S)$ and $S^{\prime}$ be a subset of $S$ made up of points covered by $T$. Then $\sum(S, x)=\sum\left(S^{\prime}, x\right)+$ $\sum\left(S-S^{\prime}, x\right) \geq \sum\left(S^{\prime}, x\right)$ because $\sum\left(S-S^{\prime}, x\right) \geq 0$ in virtue of condition 1. Clearly, $\sum\left(S^{\prime}, x\right)$ is the sum of $x_{i}+x_{j}$ over all $P_{i} P_{j} \in T$. Since $x_{i}+x_{j} \geq C_{i j}$ by condition 2 , we arrive at the inequality $\sum\left(S^{\prime}, x\right) \geq \sum(T, C)$, which implies that $\sum(S, x) \geq v_{C}(S)$.

Condition 3 in Theorem 5.3 can be replaced by a weaker condition $3^{\prime} x_{1}+\cdots+x_{n} \leq$ $v_{C}(N)$ because conditions 1 and 2 imply that $x_{1}+\cdots+x_{n} \geq v_{C}(N)$. Conditions 1 and $3^{\prime}$ amount to the assumption that $x$ is a payoff vector. Thus, Theorem 5.3 can also be stated as the following equivalence: a payoff vector $x$ is in the core if and only if $x_{i}+x_{j} \geq C_{i j}$ for any network line $P_{i} P_{j}$.

To solve a system of inequalities is a more difficult task than to solve a system of equations. Therefore, the problem of whether the network game has a nonempty core and what payoff vectors are in there would be easier to cope with if one could replace with equations at least some of the inequalities of the form $x_{i}+x_{j} \geq C_{i j}$. It turns out that equations $x_{i}+x_{j}=C_{i j}$ and sharp inequalities $x_{i}+x_{j}>C_{i j}$ occur in two distinct types of lines in $L$ : network-optimal and network-suboptimal. 
A line $P Q$ in $L$ is called network-optimal if $P Q \in T$ for some network-optimal matching $T$. Let $L^{o}$ denote the subset of $L$ which consists of network-optimal lines. Networksuboptimal lines are the elements of $L-L^{o}$. Since for homogeneous networks the terms "network-optimal matching" and "optimal matching" mean the same, the term "optimal line" will be used instead of "network-optimal line" in this case.

THEOREM 5.4. If a payoff vector $x$ is in the core of the game $\left(N, v_{C}\right)$ and $P_{i} P_{j}$ is a network line, then $x_{i}+x_{j}=C_{i j}$ if and only if $P_{i} P_{j}$ is network-optimal.

Theorem 5.4 which is a trivial consequence of Theorem 4.1 shows which lines are most likely to be used for bilateral transactions if the game associated with a one-exchange network $C$ has a nonempty core.

\section{A graph-theoretic criterion for the existence of a nonempty core in a homo-} geneous generalized assignment game. Henceforth we consider only homogeneous one-exchange networks. Since the choice of the constant $C$ is inessential, we put $C=1$, and write $v_{G}$ instead of $v_{C}$ to mark that the characteristic function game is now determined uniquely by $G=(N, L)$ according to the formula: $v_{G}(S)=\beta_{1}\left(G_{S}\right)$.

We show in this section that the existence of a nonempty core for the game $\left(N, v_{G}\right)$ depends on two structural parameters of $G$, defined in Section 1, the line independence number $\beta_{1}(G)$ and point covering number $\alpha_{0}(G)$.

Let $T$ be an optimal matching. To cover all lines in $L$, one needs to pick at least one point from each line in $T$, and possibly add some points taken from the lines in $L-T$. Therefore, $\alpha_{0}(G) \geq \beta_{1}(G)$. If $\alpha_{0}(G)=\beta_{1}(G), G$ is said to have a line-core (the line-core itself is defined as the union of all matchings $T$ such that $|T|=\alpha_{0}(G)$; see [8], 98).

We are now in a position to prove the following sufficient condition for the existence of a nonempty core for the Bienenstock-Bonacich game.

THEOREM 6.1. If $\beta_{1}(G)=\frac{1}{2} n$ or $\beta_{1}(G)=\alpha_{0}(G)$, then the game associated with $a$ homogeneous one-exchange network over $G$ has a nonempty core.

Proof. If $v_{G}(N)=\beta_{1}(G)=\frac{1}{2} n$, we put $x_{i}=\frac{1}{2}$ for all $i$. Since all three conditions in Theorem 5.3 are met, the payoff vector so defined is in the core. Assume now that $\beta_{1}(G)=\alpha_{0}(G)$. Let $S$ be a minimum point cover for $G$, that is, $|S|=\alpha_{0}(G)$. Put $x_{i}=1$ for all $P_{i} \in S$ and $x_{i}=0$ for all $P_{i} \in N-S$. We will show that $x \in \operatorname{Cr}(v G)$. Clearly, $x_{i} \geq 0$ for all $P_{i} \in N$ so that condition 1 in Theorem 5.3 is satisfied. Since $\sum(N, x)=|S|=\alpha_{0}(G)=\beta_{1}(G)=v_{G}(N)$, condition 3 holds as well. To complete the proof one needs to show that $x_{i}+x_{j} \geq 1$ for every line $P_{i} P_{j} \in L$. Since $S$ is a point cover for $G$, for every line $P_{i} P_{j} \in L$ we have $P_{i} \in S$ or $P_{j} \in S$. Therefore, $x_{i}=1$ or $x_{j}=1$, and $x_{i}+x_{j} \geq 1$.

Theorem 6.1 gives two different sufficient conditions for the existence of the core: $\beta_{1}(G)=\frac{1}{2} n$ and $\beta_{1}(G)=\alpha_{0}(G)$. The complete 4-node graph $K_{4}$ satisfies the first condition but not the second $\left(\beta_{1}\left(K_{4}\right)=2\right.$ and $\left.\alpha_{0}\left(K_{4}\right)=3\right)$. $B_{1}-A-B_{2}$ satisfies the second condition but not the first. In many graphs with even number of nodes both hold true, the Dyad $A_{1}-A_{2}$ being the simplest example. 
The union $\beta_{1}(G)=\frac{1}{2} n$ or $\beta_{1}(G)=\alpha_{0}(G)$ of two conditions is not necessary for the existence of the core. However, there are only 8 one-exchange networks with at most 8 nodes for which both conditions are not met and the associated game has a nonempty core. These networks are obtained by connecting the central node in a 2-Star or 3-Star with one or more nodes in $K_{4}$. A $k$-Star is a graph whose line set has the form $\left\{A B_{i}: i=1, \ldots, k\right\}$.

Theorem 6.1 implies that every homogeneous assignment game has a nonempty core. To demonstrate this, one needs a non-trivial theorem proved by König (Theorem 10.2 in [8]) which states that $\beta_{1}(G)=\alpha_{0}(G)$ for any bipartite graph $G$. However, Shubik and Shapley's ([10]) existence theorem is stronger than our corollary as it applies to all assignment games, not only homogeneous.

Following Bonacich ([2]) we consider for every graph $G=(N, L)$ its subgraph $G^{o}=\left(N, L^{o}\right)$ whose line set $L^{o}$ consists of all optimal lines in $G$. If $G^{o}$ is connected, that is, any two distinct points are joined by a chain made up of lines in $L^{o}$, we say that the one-exchange network over $G$ is game-indecomposable.

THEOREM 6.2. If the homogeneous one-exchange network over $G$ is game-indecomposable, then the core of the associated game $\left(N, v_{G}\right)$ is not empty if and only if $\beta_{1}(G)=\frac{1}{2} n$ or $\beta_{1}(G)=\alpha_{0}(G)$.

Proof. We have already proven (Theorem 6.1 ) that condition $\beta_{1}(G)=\frac{1}{2} n$ or $\beta_{1}(G)=$ $\alpha_{0}(G)$ suffices for the existence of a nonempty core for any $G$. Assume now that $G$ is game-indecomposable. To prove necessity, assume that $x \in \operatorname{Cr}\left(v_{G}\right)$ for some $x$. Consider an optimal matching $T$ in $G$. Suppose that $\beta_{1}(G)<\frac{1}{2} n$. The proof of Theorem 6.2 will be completed if we show that $\alpha_{0}(G)=\beta_{1}(G)$. Since $|T|<\frac{1}{2} n$, there exists a point $P_{i}$ not covered by $T$. Theorem 5.1 implies that $x_{i}=0$. The connectedness of $G^{o}=\left(N, L^{o}\right)$ means that for every $P_{j} \neq P_{i}$ there exists a chain from $P_{i}$ to $P_{j}$ made up of optimal lines. It follows from Theorem 5.4 that the $x$ values assigned to the endpoints of any line in this chain sum up to 1 . As a consequence, $x_{h}=0$ or $x_{h}=1$ for all $P_{h} \in N$.

We define the set of points $S$ by means of the condition $P_{j} \in S$ if $x_{j}=1$ and $P_{j} P_{k} \in T$ for some $P_{k}$. Let $S^{\prime}$ be the set of the remaining points which occur in lines in $T$. Since for every line $P_{j} P_{k} \in T$ we have $x_{j}+x_{k}=1$, either $P_{j}$ is in $S$ and $P_{k}$ is in $S^{\prime}$ or conversely. Therefore, $|S|=|T|=\beta_{1}(G)$. To derive the conclusion that $\alpha_{0}(G)=\beta_{1}(G)$, we must only show that $S$ is a point cover for $G$.

Suppose for an indirect proof that $P_{i} \notin S$ and $P_{j} \notin S$ for some $P_{i} P_{j} \in L$. $P_{i} \notin S$ implies that $P_{i} \in S^{\prime}$ or $P_{i} \in N-\left(S \cup S^{\prime}\right)$. If $P_{i} \in S^{\prime}$, then $x_{i}=0$. If $P_{i} \in N-\left(S \cup S^{\prime}\right)$, then $x_{i}=0$ as well, which results from Theorem 5.1 and the fact that $P_{i}$ is game-excludable as a point not covered by the optimal matching $T$. Similarly, $P_{j} \notin S$ implies that $x_{j}=0$. The conclusion that $x_{i}+x_{j}=0$ contradicts the inequality $x_{i}+x_{j} \geq 1$ which must hold for every $P_{i} P_{j} \in L$ according to condition 2 in Theorem 5.3. The proof of Theorem 6.2 is completed.

7. Decomposition of a homogeneous one-exchange network into gamecomponents. Consider now an arbitrary homogeneous one-exchange network over $G=(N, L)$. If $G^{o}=\left(N, L^{o}\right)$ is not connected, then there exists a partition of $N$ into 
pairwise disjoint nonempty subsets $N_{1}, \ldots, N_{m}$ such that all subgraphs $G_{i}^{o}=G_{N_{i}}^{o}$ of $G^{o}$ generated by the $N_{i}$ are connected. The subgraphs $G_{i}=G_{N_{i}}$ of $G$ generated by the same subsets of $N$ will be called game-components of $G$. In general, a component of an unconnected graph may reduce to an isolated point. This is not possible for $G^{o}$.

THEOREM 7.1. Each game-component of a homogeneous one-exchange network has at least two nodes.

Proof. Suppose that a single point $P_{i}$ forms a game component. Since $G$ is connected, $P_{i}$ must be tied to a point $P_{j}$ in another game component by a suboptimal line $P_{i} P_{j} . P_{j}$ does not form another single point game-component for otherwise line $P_{i} P_{j}$ could be added to any optimal matching in $G$, which is impossible. Thus, $P_{j}$ lies within a game-component with at least two points and there exists an optimal matching $T$ covering $P_{j}$. By replacing the line in $T$ which covers $P_{j}$ with $P_{i} P_{j}$ we obtain a matching with the same number of lines as $T$. Hence line $P_{i} P_{j}$ is optimal, which is a contradiction.

Theorem 7.1 is important due to the corollaries one can derive from it. First, all hanging lines are optimal. $P Q$ is a hanging line if $\operatorname{deg}(P)=1$ and $\operatorname{deg}(Q)>1$ or $\operatorname{deg}(P)>1$ and $\operatorname{deg}(Q)=1$.

The second corollary is that every $P$ is covered by an optimal matching. In other words, every player can contribute to the maximization of group payoff.

In any superadditive game $(N, v)$, the players can be divided into three categories with respect to how the maximization of the group payoff depends on each player. The least "powerful" players are those who are not members of any minimal coalition $S$ such that $v(S)=v(N)$. The second set consists of those players who participate each in at least one minimal coalition but not in all minimal coalitions with this property. The third set is formed by the players who are members of every minimal coalition maximizing the group payoff. The game associated with a homogeneous one-exchange network may have only the second and third type of players. This distinction is exactly that between game-excludable and game-nonexcludable positions.

To each game component $G_{i}=\left(N_{i}, L_{i}\right)$ of a connected graph $G=(N, L)$ there corresponds the Bienenstock-Bonacich game $\left(N_{i}, v_{G_{i}}\right)$. It is not difficult to verify that $v_{G_{i}}$ coincides with the restriction of $v_{G}$ to the subsets of $N_{i}$.

If $T$ is an optimal matching in $G$, then $T \cap L_{i}$ is an optimal matching in $G_{i}$. Hence, each optimal line in $G$ located in $G_{i}$ is optimal in $G_{i}$. On the other hand, the union of optimal matchings taken from all game-components is an optimal matching in $G$. Therefore, a line in $L_{i}$ is optimal in $G_{i}$ if and only if it is optimal in $G$. The type of a position in a game-component coincides with its type in the whole network. For any point $P$ in $G_{i}, P$ is game-(non)excludable in $G_{i}$ if and only if $P$ is game-(non)excludable in $G$. Theorems 7.2 and 7.3 are simple consequences of these facts.

THEOREM 7.2. The game-components of a homogeneous one-exchange network are gameindecomposable.

THEOREM 7.3. If $G_{1}, \ldots, G_{m}$ are game-components of $G$, then $v_{G}(N)=\sum_{i} v_{G_{i}}\left(N_{i}\right)$, or, in graph-theoretic terms, $\beta_{1}(G)=\sum_{i} \beta_{1}\left(G_{i}\right)$. 
The game-power of position $P$ over $Q$ is defined by the condition: there exists an optimal matching which covers $P$ and does not cover $Q$ ( $P$ can game-exclude $Q$ ) and every optimal matching which covers $Q$ covers $P(Q$ cannot game-exclude $P)$. Theorem 7.4 implies that the dyads in game- power relation can be found only inside game-components.

TheOREM 7.4. A game-excludable point in $G$ can be tied only to points in the same game-component of $G$.

Proof. Consider two points $P$ and $Q$ in two distinct game-components $G_{i}$ and $G_{j}$. If both $P$ and $Q$ are game-excludable, then $P$ is game-excludable in $G_{i}$ and $Q$ is game-excludable in $G_{j}$. This implies the existence of an optimal matching $T$ in $G$ covering neither $P$ nor $Q$. If line $P Q$ were in $G$, it could be added to $T$, and $T$ would not be optimal. If $P$ is game-excludable and $Q$ is game-nonexcludable, we consider an optimal $T$ which covers $Q$ and does not cover $P$. If line $P Q$ were in $G$, then the line in $T$ covering $Q$ in $G_{j}$ could be replaced with $P Q$ to obtain from $T$ a matching $T^{\prime}$ containing $P Q$ and having as many lines as $T$. Then line $P Q$ would be optimal, but all lines joining points in distinct components are suboptimal.

We are now in a position to demonstrate the second main theorem which together with Theorem 6.2 provides a complete graph-theoretic characterization of one-exchange networks for which the Bienestock-Bonacich game has a nonempty core.

THEOREM 7.5. The game $\left(N, v_{G}\right)$ associated with a homogeneous one-exchange network over $G=(N, L)$ has a nonempty core if and only all games $\left(N_{i}, v_{G_{i}}\right)$ associated with game-components $G_{i}=\left(N_{i}, L_{i}\right)$ of $G$ have nonempty cores.

Proof. Assume that the payoff vector $x$ is in the core of $\left(N, v_{G}\right)$. Theorem 7.3 implies that $v_{G}(N)=\sum_{i} v_{G_{i}}\left(N_{i}\right)=\sum_{i} v_{G}\left(N_{i}\right)$. Thus, the coalition structure $\left\{N_{1}, \ldots, N_{m}\right\}$ is optimal and $x$ is feasible for it. As a consequence, the sum of $x_{j}$ over all $P_{j}$ in $N_{i}$ equals $v_{G}\left(N_{i}\right)=v_{G_{i}}\left(N_{i}\right)$. The $x$ payoffs which go to the players in $N_{i}$ satisfy (group rationality) condition 3 in Theorem 5.3 with respect to $\left(N_{i}, v_{G_{i}}\right)$. Clearly, conditions 1 (individual rationality) and 2 (dyadic rationality) are also met for any game $\left(N_{i}, v_{G_{i}}\right)$.

Assume in turn that every game $\left(N_{i}, v_{G_{i}}\right)$ has a nonempty core. We show how to construct a payoff vector that will be in the core of $\left(N, v_{G}\right)$ through selecting a certain payoff vector from $\operatorname{Cr}\left(v_{G_{i}}\right)$. Since the core payoffs allotted to the players in each $N_{i}$ sum up to $v_{G_{i}}\left(N_{i}\right)$, the overall payoff sum equals $v_{G}(N)$ by Theorem 7.3. Therefore, any payoff vector constructed in such a way satisfies the group rationality condition. Clearly, the individual rationality condition is also met. It remains to be shown that core payoffs in each component can be chosen so that the dyadic rationality condition is met for any $P$ and $Q$ such that $P Q \in L, P \in N_{i}, Q \in N_{j}$ where $G_{i}=\left(N_{i}, L_{i}\right)$ and $G_{j}=\left(N_{j}, L_{j}\right)$ are two distinct game-components of $G$. If $\beta_{1}\left(G_{i}\right)=\frac{1}{2} n_{i}$ where $n_{i}=\left|N_{i}\right|$, then the payoff vector such that $x_{P}=\frac{1}{2}$ for any $P \in N_{i}$ is in the core of $\left(N_{i}, v_{G_{i}}\right)$. If $\beta_{1}\left(G_{i}\right)<\frac{1}{2} n_{i}$, then $2 \beta_{1}\left(G_{i}\right)<n_{i}$ so that $N_{i}$ must contain at least one point $P$ not covered by a matching $T$ in $G_{i}$ such that $|T|=\beta_{1}\left(G_{i}\right)$. Then, for any $P \in N_{i}, x_{P}=0$ or $x_{P}=1$ for any payoff vector in the core of the game $\left(N_{i}, v_{G_{i}}\right)$. If $P Q \in L$ where $Q \in N_{j}$, then the case $x_{P}=0$, or the case where $P$ is game-excludable, is impossible in virtue of Theorem 7.4. We conclude that one can always find a payoff vector in $\operatorname{Cr}\left(v_{G_{i}}\right)$ such that $x_{P} \geq \frac{1}{2}$ for any $P$ in $N_{i}$ 
connected to a $Q$ in $N_{j}$, which implies in turn that $x_{P}+x_{Q} \geq 1$ for any line $P Q \in L$ joining two points from distinct game-components of $G$. The proof of Theorem 7.5 is completed.

For odd $n$, coreless networks occur fairly frequently in the set of $n$-node connected graphs. To determine the frequencies, given below with the numbers of all connected $n$-node graphs, I wrote a computer program for which I used as input the list of adjacency matrices (generated by John Skvoretz, see [12]) of all nonisomorphic connected graphs with the number of nodes ranging from 2 to 8 .

\begin{tabular}{|r|r|r|r|r|r|r|}
\hline 2 & 3 & 4 & 5 & 6 & 7 & 8 \\
\hline 0 & 1 & 0 & 12 & 3 & 626 & 279 \\
1 & 2 & 6 & 21 & 112 & 853 & 11117 \\
\hline
\end{tabular}

According to Theorem 7.5, to check if a one-exchange network is coreless, one has to decompose it into game-components and verify if each component satisfies the necessary and sufficient condition given in Theorem 6.2. Only 15 out of 921 coreless networks with up to 8 nodes have 2 game-components, all other are game-indecomposable of which 797 have no suboptimal lines.

The construction of the core for the whole network from the cores of particular gamecomponents entails imposing additional constraints on the payoffs in each component. One can illustrate this using the simplest homogeneous one-exchange network with two game components, namely, the 4-Chain network $B_{1}-A_{1}-A_{2}-B_{2}$.

Any payoff vector $\left(a_{1}, 1-a_{1}\right)$, where $a_{1}$ and $1-a_{1}$ are the payoffs of $A_{1}$ and $B_{1}$, is in the core of the first game-component for any $a_{1}$ from the $[0,1]$ interval. Similarly, the core of the second game-component consists of payoff vectors of the form $\left(a_{2}, 1-a_{2}\right)$. The combination of two vectors $\left(a_{1}, 1-a_{1}\right)$ and $\left(a_{2}, 1-a_{2}\right)$ is in the core of the 4-Chain game if and only if $a_{1}$ and $a_{2}$ satisfy the inequality $a_{1}+a_{2} \geq 1$. With this restriction the square $[0,1] \times[0,1]$ shrinks to the triangle with vertices $(0,1,(1,0),(1,1)$.

\section{References}

[1] E. J. Bienenstock and P. Bonacich, The core as a solution to exclusionary networks, Social Networks 14 (1992), 231-243.

[2] P. Bonacich, A behavioral foundation for a structural theory of power in exchange networks, Social Psychology Quarterly 61 (1998), 185-198.

[3] P. Bonacich, An algebraic theory of strong power in negatively connected exchange networks, Journal of Mathematical Sociology 23 (1999), 203-224.

[4] E. J. Bienenstock and P. Bonacich, Assignment games, chromatic number, and exchange theory, Journal of Mathematical Sociology 17 (1993), 243-259.

[5] E. J. Bienenstock and P. Bonacich, When rationality fails. Unstable exchange networks with empty core, Rationality and Society 2 (1995), 293-320.

[6] E. J. Bienenstock and P. Bonacich, Latent classes in exchange networks: sets of positions with common interests, Journal of Mathematical Sociology 22 (1997), 1-28. 
[7] N. E. Friedkin, An expected value model of social power: predictions for selected exchange networks, Social Networks 14 (1992), 213-229.

[8] F. Harary, Graph Theory, Addison-Wesley, Reading, MA, 1969.

[9] G. Owen, Game Theory, 3rd edition, Academic Press, New York-London, 1995.

[10] L. S. Shapley and M. Shubik, The assignment game I: the core, International Journal of Game Theory 1 (1972), 111-130.

[11] M. Shubik, A Game-Theoretic Approach to Political Economy, The MIT Press, Cambridge, MA, 1984.

[12] J. Skvoretz, An algorithm to generate connected graphs, Current Research in Social Psychology 1(1996) no. 5, http://www.uiowa.edu/ grpproc/crisp/crisp.1.5.html

[13] T. Sozański, The Mathematics of Exchange Networks, Part II (Chapter 3: Exclusion and power; Chapter 4: The principle of equal dependence; Chapter 5: Game theory and exchange networks), in process, draft available since August 2004 at the author's homepage http://www.cyf-kr.edu.pl/ ussozans/.

[14] D. Willer (ed.), Network Exchange Theory, Praeger, Westport, Ct.-London, 1999. 